\title{
Ansiedade e consumo de ansiolíticos entre estudantes de enfermagem de uma universidade pública
}

\author{
Anxiety and the consumption of anxiolytics among nursing students of a public university
}

Ansiedad y consumo de ansiolíticos entre estudiantes de enfermería de una universidad pública

Katia Colombo Marchi ${ }^{1}$, Alessandra Marino Bárbaro², Adriana Inocenti Miasso ${ }^{3}$, Carlos Renato Tirapelli ${ }^{4}$

${ }^{1}$ Enfermeira, Mestre em Enfermagem Psiquiátrica. Discente do Programa de Pós-Gradação em Farmacologia, nível Doutorado, da Faculdade de Medicina de Ribeirão Preto da Universidade de São Paulo (USP). Ribeirão Preto, SP, Brasil. E-mail: katia.marchi@usp.br.

2 Enfermeira. Discente do Programa de Pós-Graduação em Enfermagem Psiquiátrica, nível Mestrado, da Escola de Enfermagem de Ribeirão Preto (EERP) da USP. Ribeirão Preto, SP, Brasil. E-mail: allebarbaro@hotmail.com.

${ }^{3}$ Enfermeira, Doutora em Enfermgem. Professora Doutora da EERP/USP. Ribeirão Preto, SP, Brasil. E-mail: amiasso@eerp.usp.br.

${ }^{4}$ Farmacêutico, Doutorado em Farmacologia. Professor Associado da EERP/USP. Ribeirão Preto, SP, Brasil. E-mail: crtirapelli@eerp.usp.br.

\section{RESUMO}

Trata-se de estudo transversal, descritivo, de abordagem quantitativa realizado em uma escola pública de enfermagem do estado de São Paulo, que avaliou a incidência de ansiedade entre os estudantes, seu conhecimento sobre os ansiolíticos e o padrão de consumo destes medicamentos. Dos 308 estudantes, $16 \%$ estavam utilizando ansiolíticos no período em que a pesquisa foi realizada ou já usaram em algum momento de suas vidas; destes, 35\% apresentaram ansiedade severa, de acordo com a aplicação do Inventário de Ansiedade de Beck. Mesmo tendo recebido orientação, a maioria dos usuários referiram possuir dúvidas, especialmente no que diz respeito à interação com outros fármacos, efeitos colaterais e seu potencial para causar dependência. Destaca-se a importância do reconhecimento e tratamento da ansiedade entre os estudantes, uma vez que sua presença, quando em níveis mais altos, pode afetar de forma significativa a formação da identidade do estudante e sua qualidade de vida.

Descritores: Ansiedade; Estudantes de Enfermagem; Ansiolíticos; Psicotrópicos.

\section{ABSTRACT}

This cross-sectional, descriptive study was performed at a public nursing school in the state of São Paulo, using a quantitative approach. An evaluation was made of the incidence of anxiety among the students, their knowledge regarding anxiolytics and the consumption pattern of those drugs. Of the 308 students, $16 \%$ was using anxiolytics when the study was performed or had already used anxiolytics at some time in their lives; $35 \%$ of the later suffered from severe anxiety according to the application of the Beck's Anxiety Inventory. Despite the guidance they received, most users referred having doubts mainly regarding the interaction of anxiolytics with other drugs, their side effects and their potential of being addictive. It is highlighted that recognizing among students and offering treatment is important, as its presence, when at high level can have a significant effect on the development of the student's identity and his/her quality of life.

Descriptors: Anxiety; Students, Nursing; Anti-Anxiety Agents.

\section{RESUMEN}

Estudio transversal, descriptivo, de abordaje cuantitativo, realizado en una escuela pública de enfermería del estado de São Paulo, que evaluó la incidencia de ansiedad entre los estudiantes, su conocimiento sobre ansiolíticos y el patrón de consumo de tales medicamentos. De los 308 estudiantes, $16 \%$ estaba utilizando ansiolíticos durante el período de la realización del estudio, o ya los usaron en algún momento de sus vidas. Entre ellos, 35\% presentaban ansiedad severa, de acuerdo a la aplicación del Inventario de Ansiedad de Beck. Inclusive habiendo recibido orientaciones, la mayoría de los usuarios refirió dudas, especialmente respecto a la interacción con otros fármacos, efectos colaterales y su potencial para causar dependencia. Se destaca la importancia de reconocimiento y tratamiento de la ansiedad entre los estudiantes, toda vez que su presencia en niveles elevados puede afectar significativamente la formación de la identidad del estudiante y su calidad de vida.

Descriptores: Ansiedad; Estudiantes de Enfermería; Ansiolíticos. 


\section{INTRODUÇÃO}

De uma maneira geral, os transtornos de ansiedade têm aumentado expressivamente no último século, sobretudo devido às profundas transformações ocorridas no âmbito econômico e cultural que foram acompanhadas por pressões de uma sociedade moderna, tecnológica e principalmente cada vez mais competitiva ${ }^{(1)}$.

A vida de estudantes universitários conta com diversos fatores ansiogênicos. A apreensão em relação ao futuro é um componente contínuo que contribui significantemente para o desenvolvimento da ansiedade nesse grupo(2). Da mesma forma, o período universitário, ainda que traga sentimentos positivos e de alcance de uma meta programada, por vezes pode se tornar um período crítico e de maior vulnerabilidade ${ }^{(3)}$.

Estudo demonstra que, ao ingressarem na universidade, os estudantes são submetidos a uma grande carga de estresse, principalmente devido à cobranças pessoais, expectativas familiares, encargos financeiros, convivência com colegas de quarto, atividades domésticas, horários desordenados de sono e longas horas de estudo(4). Além disso, transformações maturacionais (fisiológicas, neurológicas e psicológicas) decorrentes da transição entre a fase de adolescente e a de adulto, levam os estudantes a vivenciarem um período de crise, por exigir a adaptação a um novo papel social(1).

Enquanto níveis moderados de ansiedade são necessários para a aprendizagem, níveis mais elevados, por sua vez, impedem que ela ocorra ${ }^{(5)}$. Isto se deve pela interferência negativa sobre alguns aspectos cognitivos do aluno, em sua atenção seletiva e codificação de informações na memória, bloqueando a compreensão e o raciocínio, reduzindo a concentração e percepção, a aquisição de habilidades intelectuais e seu desempenho em geral ${ }^{(6)}$.

Estudantes de ensino superior da área da saúde formam uma população que merece especial atenção por apresentarem maior nível de ansiedade, quando comparados a outras áreas de ensino ${ }^{(4)}$. Estes, incluindo os estudantes de enfermagem, possuem durante o período de graduação alguns fatores a mais que causam ansiedade. A experiência da prática clínica, o lidar com o ser humano, o contato com o sofrimento psíquico, a observação constante dos instrutores no cenário da prática, o medo de cometer erros, sentimentos de inadequação e falta de inclusão em grupos de pessoal clínico são destacados na literatura como os principais desencadeantes ${ }^{(5)}$.

Também é importante salientar que estes estudantes lidam, muitas vezes, com a situação altamente estressante da iminência de morte ${ }^{(7)}$. Tais situações exaustivas trazem consigo um conflito e a exigência de um esforço de ajustamento e adaptação maior, surgindo, então, a necessidade de traçar estratégias que possibilitem a superação dessa situação. Neste contexto, a busca por essa adaptação leva o indivíduo a procurar ajuda, sendo frequente o início do uso de psicofármacos, muitas vezes de forma indevida, fato que estabelece mais um problema relevante nas sociedades contemporâneas(8-10).

Diversas modalidades de tratamento tanto psicoterápicas quanto farmacológicas vêm sendo utilizadas no tratamento dos transtornos de ansiedade. Os benzodiazepínicos estão entre os fármacos mais prescritos no mundo e são utilizados principalmente como ansiolíticos e hipnóticos, além de possuir ação miorrelaxante e anticonvulsivante ${ }^{(11)}$. Uma grande preocupação quanto ao uso dos benzodiazepínicos é seu potencial рага o abuso, dependência, abstinência, tolerância, sedação, prejuízos psicomotores e sua interação com o álcool ou outras drogas hipnóticas. É importante ressaltar que o álcool é um contribuinte comum para as mortes envolvendo o uso de benzodiazepínicos ${ }^{(12)}$. A orientação médica e o consequente conhecimento por parte do paciente sobre os efeitos colaterais dos benzodiazepínicos estão distantes de ser o ideal, porém a orientação é um fator muito importante para minimizar a incidência de erros e efeitos colaterais, aumentar a adesão e efetividade da terapia ${ }^{(11)}$.

Nesse sentido, considerando o aumento da prevalência de ansiedade entre os estudantes universitários, as consequências do tratamento inadequado desta patologia e a escassez de estudos na literatura sobre tal temática, este estudo teve como objetivos analisar a presença da ansiedade em estudantes de enfermagem de uma universidade pública do estado de São Paulo, avaliar o conhecimento dos estudantes que utilizam fármacos com ação ansiolítica e caracterizar o padrão de consumo desses medicamentos. 


\section{MÉTODOS}

Trata-se de estudo transversal e descritivo de abordagem quantitativa realizado em uma escola pública de enfermagem do estado de São Paulo no período de agosto de 2010 a junho de 2011. Esta instituição possui quatro turmas de bacharelado e cinco de licenciatura, totalizando 568 alunos, que foram convidados a participar do estudo. Destes, 308 aceitaram respondendo aos questionários.

O primeiro questionário aplicado consiste em quatro partes. Na primeira parte, foram coletados dados de identificação dos sujeitos; na segunda, o objetivo foi avaliar se o usuário de ansiolítico considerava importante que fosse feita alguma orientação sobre o uso do medicamento; na terceira parte foram coletados dados a respeito do conhecimento do usuário sobre o medicamento ansiolítico e na última parte do questionário foi caracterizado o padrão de consumo dos ansiolíticos (tipo, frequência de consumo, acompanhamento médico e motivo do consumo).

Também foi aplicado aos 308 alunos o Inventário de Ansiedade de Beck (Beck Anxiety Inventory - BAI). O BAI é um questionário de auto-relato utilizado para medir a gravidade da ansiedade de um indivíduo. Consiste em 21 questões, cada uma com quatro possíveis respostas, sobre como o indivíduo tem se sentido na última semana, expressas em sintomas comuns de ansiedade (como sudorese e sentimentos de angústia). Possui um resultado máximo de 63 e as categorias são: 0-7: grau mínimo de ansiedade; 8-15: ansiedade leve; 16-25 ansiedade moderada; 26-63: ansiedade grave(13-14). A técnica de auto-relato foi utilizada para coleta de dados e as informações foram registradas pelos próprios sujeitos da pesquisa. Destaca-se que o pesquisador permaneceu com os estudantes e esclareceu as dúvidas dos mesmos durante o preenchimento dos instrumentos.

Os dados foram analisados no programa Statistical Package for the Social Science (SPSS, versão 17.0). Foram investigadas associações estatísticas entre as variáveis dependentes e independentes usando o teste Quiquadrado $\left(x^{2}\right)$, sendo a hipótese de associação aceita quando $p$ encontrado for menor ou igual a 0,05.

O projeto foi aprovado pelo Comitê de Ética e Pesquisa envolvendo seres humanos da Escola de Enfermagem de Ribeirão Preto (protocolo 1181/2010). A aplicação do questionário e do BAl foi procedida após a anuência e assinatura do Termo de Consentimento Livre e Esclarecido pelos sujeitos da pesquisa (CNS 196/96).

\section{RESULTADOS}

Dos 308 estudantes que participaram do estudo, $16 \%$ ( $n=48$ ) estavam utilizando ansiolíticos no período em que a pesquisa foi realizada ou já utilizaram em algum momento de suas vidas. Destes, $35 \%$ eram do curso de bacharelado e $64 \%$ do curso de licenciatura em enfermagem. Os dados socioeconômicos dos participantes estão demonstrados na Tabela 1. 
Tabela 1: Frequência e porcentagem das características sociodemográficas dos estudantes de enfermagem que estavam usando ou já haviam utilizado ansiolíticos. Ribeirão Preto, SP, 2011.

\begin{tabular}{|c|c|c|}
\hline Variáveis & $F(n=48)$ & $\%$ \\
\hline \multicolumn{3}{|l|}{ Programa } \\
\hline Bacharelado & 17 & 35,4 \\
\hline Licenciatura & 31 & 64,5 \\
\hline \multicolumn{3}{|l|}{ Gênero } \\
\hline Masculino & 7 & 14,6 \\
\hline Feminino & 41 & 85,4 \\
\hline \multicolumn{3}{|l|}{ Idade } \\
\hline $18-22$ anos & 27 & 56,3 \\
\hline 23-27 anos & 14 & 29,2 \\
\hline 28 anos ou mais & 7 & 14,6 \\
\hline \multicolumn{3}{|l|}{ Estado civil } \\
\hline Solteiro & 42 & 87,5 \\
\hline Casado & 5 & 10,4 \\
\hline Outros & 1 & 2,1 \\
\hline \multicolumn{3}{|l|}{ Número de filhos } \\
\hline Sem filho & 44 & 91,7 \\
\hline 1 filho & 0 & 0 \\
\hline 2-3 filhos & 4 & 8,3 \\
\hline \multicolumn{3}{|l|}{ Trabalha } \\
\hline Sim & 14 & 29,2 \\
\hline Não & 34 & 70,8 \\
\hline \multicolumn{3}{|l|}{ última consulta médica } \\
\hline Menos 3 meses & 25 & 52,1 \\
\hline 4 a 6 meses & 14 & 29,2 \\
\hline 7 meses a 1 ano & 8 & 16,7 \\
\hline Mais que 1 ano & 1 & 2,1 \\
\hline
\end{tabular}

Na Tabela 2 é possível observar que destes 48 estudantes, a maioria recebeu orientações sobre os efeitos colaterais e interações medicamentosas dos ansiolíticos $\left(x^{2}{ }_{1}=12,0 ; p<0,05\right)$. A orientação foi realizada majoritariamente pelo médico $\left(x^{2}{ }_{1}=82,3 ; p<0,05\right)$, sendo citado uma vez o enfermeiro. A maioria dos participantes $(93,8 \%)$ considerou a orientação importante $\left(x^{2}{ }_{1}=36,7\right.$; $p<0,05)$ para aumentar a confiança, segurança e efetividade da terapia, minimizar os efeitos colaterais e interações medicamentosas. Ainda que a orientação préadministração no uso de ansiolíticos fosse feita para a maioria dos participantes, não houve diferença significativa $\left(x^{2}{ }_{1}=0,75 ; \quad p>0,05\right)$ entre usuários que apresentaram $(43,8 \%)$ e os que não apresentaram dúvidas (56,3\%). As dúvidas mais frequentes foram em relação à interação de ansiolíticos com outros fármacos (62\%), efeitos colaterais (57\%), se o medicamento causa dependência (43\%), o mecanismo de ação (33\%), o tempo de tratamento necessário (28\%) e o tempo de início para a ação do fármaco (24\%). 
Tabela 2: Distribuição dos estudantes de enfermagem de acordo com a opinião sobre a importância da orientação no tratamento. Ribeirão Preto, SP, 2011.

\begin{tabular}{|c|c|c|c|}
\hline Variáveis & $F(n=48)$ & $\%$ & $x^{2}$ \\
\hline \multicolumn{4}{|l|}{ Recebeu instrução sobre uso de ansiolíticos } \\
\hline $\operatorname{Sim}$ & 36 & 75 & $x^{2}=12,0$ \\
\hline Não & 12 & 25 & $p<0,05$ \\
\hline \multicolumn{4}{|l|}{ Quem fez } \\
\hline Médico & 35 & 97,2 & $x^{2}=82,3$ \\
\hline Outro profissional & 1 & 2,8 & $p<0,05$ \\
\hline \multicolumn{4}{|l|}{ Considera a orientação importante } \\
\hline Sim & 45 & 93,8 & $x^{2}=36,7$ \\
\hline Não & 0 & 0 & $p<0,05$ \\
\hline Não sabe & 3 & 6,3 & \\
\hline \multicolumn{4}{|l|}{ Qual a importância da orientação } \\
\hline Aumenta confiança na terapia & 21 & 43,7 & \\
\hline Aumenta segurança na terapia & 21 & 43,7 & \\
\hline Aumenta efetividade da terapia & 17 & 35,4 & \\
\hline Reduz efeitos colaterais e interações com outras drogas & 19 & 39,5 & \\
\hline Outros & 0 & 0 & \\
\hline \multicolumn{4}{|l|}{ Dúvidas sobre tratamento } \\
\hline Sim & 21 & 43,8 & $x^{2}=0,75$ \\
\hline Não & 27 & 56,3 & $p>0,05$ \\
\hline
\end{tabular}

Na Tabela 3, onde estão apresentados os dados acerca do conhecimento dos estudantes sobre os ansiolíticos, deve-se ressaltar que embora $73 \%$ deles nunca tenham aumentado a dose sem consultar o médico $\left(x^{2} 1=34,6 ; p<0,05\right), 27 \%$ dos estudantes já o fez. A maioria acredita que os ansiolíticos causam tolerância, dependência e que a remoção do medicamento no final do tratamento deve ser feita de maneira gradual $\left(x^{2}{ }_{1}=32,3 ; \quad p<0,05 ; \quad x^{2}=51,1 ; \quad p<0,05 ; \quad x^{2}{ }_{1}=30,0 ; \quad p<0,05\right)$. Quando questionados sobre a presença de efeitos colaterais, $65 \%$ referiram não apresentar. Dos que apresentaram, os principais sintomas relatados foram cansaço, sonolência, distúrbios do sono, confusão mental e dores de cabeça.

Tabela 3: Distribuição dos estudantes de enfermagem de acordo com seu conhecimento acerca das ações dos ansiolíticos. Ribeirão Preto, SP, 2011.

\begin{tabular}{|c|c|c|c|}
\hline Variáveis & $F(n=48)$ & $\%$ & $x^{2}$ \\
\hline \multicolumn{4}{|l|}{ Já aumentou a dose sem consultar o médico } \\
\hline Nunca & 35 & 72,9 & $x^{2}=34,6$ \\
\hline Uma vez & 9 & 18,8 & $p<0,05$ \\
\hline Frequentemente & 4 & 8,3 & \\
\hline \multicolumn{4}{|c|}{ Está apresentando efeitos colaterais devido ao uso do ansiolítico? } \\
\hline Sim & 11 & 22,9 & $x^{2}=21,8$ \\
\hline Não & 31 & 64,6 & $p<0,05$ \\
\hline Não sabe & 6 & 12,5 & \\
\hline \multicolumn{4}{|l|}{ Os ansiolíticos podem causar dependência } \\
\hline Sim & 39 & 81,3 & $x^{2}=51,1$ \\
\hline Não & 1 & 2,1 & $p<0,05$ \\
\hline Não sabe & 8 & 16,7 & \\
\hline \multicolumn{4}{|l|}{ Os ansiolíticos podem causar tolerância } \\
\hline Sim & 33 & 68,8 & $x^{2}=32,3$ \\
\hline Não & 1 & 2,1 & $p<0,05$ \\
\hline Não sabe & 14 & 29,2 & \\
\hline \multicolumn{4}{|c|}{ A remoção do medicamento no final do tratamento deve ser feita de maneira gradual } \\
\hline Sim & 43 & 89,6 & $x^{2}=30,0$ \\
\hline Não & 0 & 0 & $p<0,05$ \\
\hline Não sabe & 5 & 10,4 & \\
\hline \multicolumn{4}{|c|}{ Você interrompeu o tratamento sem consultar o médico } \\
\hline Sim & 22 & 45,8 & $x^{2}=0,3$ \\
\hline Não & 26 & 54,2 & $p>0,05$ \\
\hline \multicolumn{4}{|l|}{ Faz uso de outros medicamentos além do ansiolítico } \\
\hline Sim & 29 & 60,4 & $x^{2}=2,0$ \\
\hline Não & 19 & 39,6 & $p>0,05$ \\
\hline
\end{tabular}


Um dado que chamou a atenção foi que aproximadamente $46 \%$ dos estudantes interromperam o tratamento sem consultar o médico $\left(x^{2}{ }_{1}=0,3 ; p>0,05\right)$. Este fato foi devido principalmente à melhora dos sintomas de ansiedade (45\%), mas também foi relatado como causas o medo da dependência, a falta de efeito do medicamento, a dificuldade para se obter nova prescrição, baixa tolerância aos efeitos colaterais e o custo do tratamento. É importante ressaltar que $60 \%$ dos estudantes referiram utilizar outras medicações além do ansiolítico, sendo os antidepressivos os mais frequentes (19\%).

Quando questionados sobre o uso de álcool durante o tratamento com ansiolíticos, 31,3\% referiram ingeri-lo, sendo que $19 \%$ o fazem aos finais de semana.

O diazepam foi o medicamento mais utilizado pelos estudantes (Gráfico 1). Vale ressaltar que foi citado pelos estudantes, quando questionados sobre os ansiolíticos que utilizam, alguns antidepressivos como a fluoxetina e a imipramina.

Gráfico 1: Frequência do uso de diferentes ansiolíticos entre estudantes de enfermagem. Ribeirão Preto, SP, 2011.

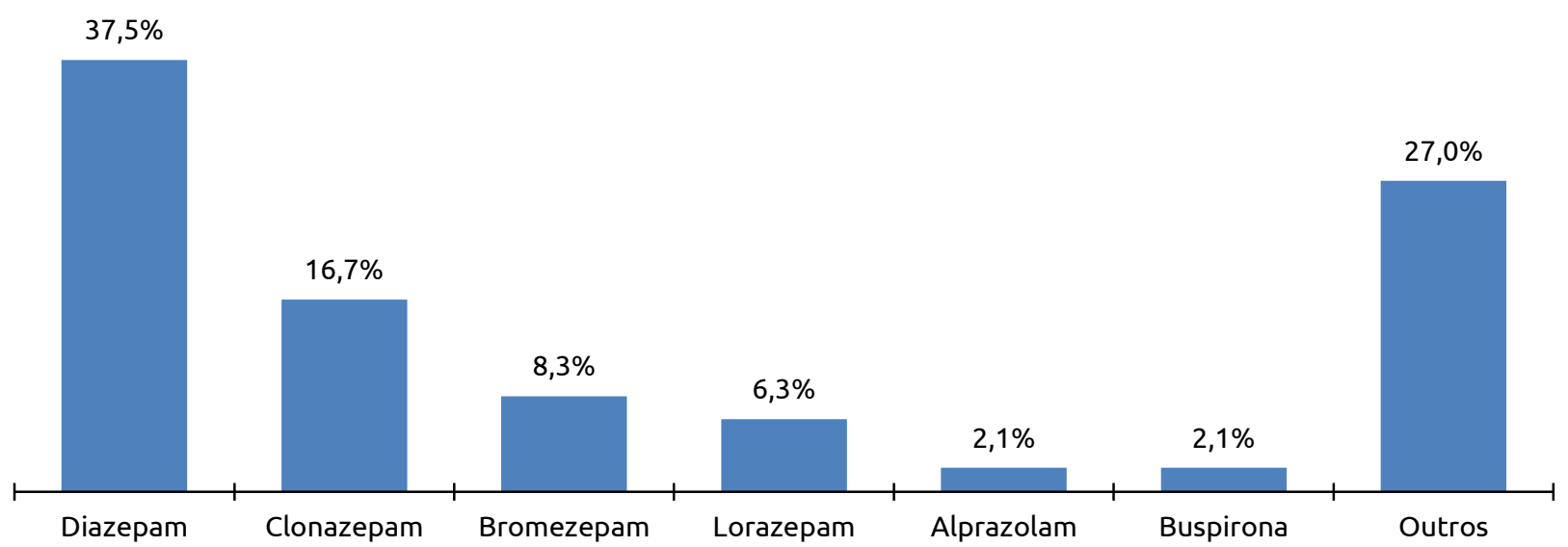

A idade com a qual o medicamento foi utilizado pela primeira vez variou de nove a 37 anos, porém com uma proporção maior aos 20 (14,6\%), 16 (12,5\%) e 18 (12,5\%) anos. Também foi constatado que $65 \%$ dos estudantes referiram possuir acompanhamento médico $\left(x^{2}{ }_{1}=3,9\right.$; $p<0,05)$. Já quando questionados sobre os principais motivos do consumo do medicamento, os mais relatados pelos alunos foram a presença de ansiedade (principalmente fobia social e síndrome do pânico), nervosismo, insônia, estresse, depressão, enxaquecas e hospitalizações.
Com a aplicação do Inventário de Ansiedade de Beck (Beck Anxiety Inventory - BAI), constatamos que dos 308 alunos avaliados, 30\% apresentou grau mínimo de ansiedade, $34 \%$ ansiedade leve, $24 \%$ moderada e $12 \%$ ansiedade considerada grave. Na amostra de estudantes que estavam utilizando ansiolíticos no período em que a pesquisa foi realizada, ou já haviam utilizado em algum momento de suas vidas, foi encontrada uma média de 22 pontos (Desvio-padrão= $\pm 11,08$ ), com $35,4 \%$ dos estudantes apresentando ansiedade grave (Tabela 4).

Tabela 4: Distribuição dos estudantes que estavam usando ou já haviam utilizado ansiolíticos de acordo com o nível de ansiedade apresentado - Inventário de Ansiedade de Beck (Beck Anxiety Inventory - BAI). Ribeirão Preto, SP, 2011.

\begin{tabular}{lcc}
\hline Níveis de ansiedade & F (n=48) & \% \\
\hline Grau mínimo de ansiedade & 4 & 8,3 \\
Ansiedade leve & 13 & 27,1 \\
Ansiedade moderada & 14 & 29,2 \\
Ansiedade severa & 17 & 35,4 \\
\hline
\end{tabular}

Não foram encontradas associações entre a presença de dúvidas e o fato dos participantes interromperem o tratamento sem consultar o médico, como também em relação ao uso de outras medicações além de ansiolíticos e a presença de efeitos colaterais $(p>0,05)$. Uma associação interessante encontrada foi que entre os estudantes com ansiedade grave, $82 \%$ não ingeriram álcool durante o tratamento com ansiolíticos, 59\% não interromperam o tratamento, porém todos já aumentaram a dose sem consultar o médico. 


\section{DISCUSSÃO}

O presente trabalho demonstrou, por meio da aplicação do Inventário de Ansiedade de Beck, que dos 308 estudantes avaliados, 34\% apresentam ansiedade leve, $24 \%$ moderada e $12 \%$ grave, ressaltando a presença de ansiedade em estudantes universitários e destacando a importância de seu reconhecimento e tratamento, uma vez que sua presença quando em níveis mais altos, pode afetar de forma significativa a formação da identidade do estudante como também sua qualidade de vida ${ }^{(15)}$.

Estudantes de enfermagem muitas vezes se sentem vulneráveis com a gestão do crescente volume de informações, o planejamento da carreira profissional, o estresse decorrente de certas características de estágios práticos, problemas relativos à qualidade do ensino e ao ambiente educacional, situações pessoais (vulnerabilidades psicológicas, situação socioeconômica, problemas familiares, situações estressantes representadas pela busca de independência e autonomia em relação aos pais, conflitos entre os trabalhos acadêmicos e lazer, conflitos ligados aos relacionamentos afetivos), além do desgaste ligado ao contato com pessoas doentes e com a morte ${ }^{(4,16-17)}$.Uma vez os fatores ansiogênicos atuando na vida do estudante, este busca uma adaptação por exceder seus recursos de enfrentamento, se tornando apreensivo, inseguro e desconfortável diante de situações que considera ameaçadoras. Desta forma, a ansiedade acaba interferindo sobre aspectos cognitivos do aluno, em sua atenção seletiva, muitas vezes bloqueando a compreensão e o raciocínio, diminuindo, assim, a aquisição de habilidades intelectuais e seu desempenho em geral(6).

Especificamente entre os estudantes que utilizam ou já utilizaram algum medicamento para a ansiedade, os resultados revelaram a presença de ansiedade moderada em $29 \%$ deles enquanto que $35 \%$ apresentaram ansiedade grave. Faz-se importante ressaltar que estudantes de enfermagem possuem um melhor desempenho no cenário tanto da prática quanto em sala de aula com um nível leve de ansiedade, porém níveis mais altos, como os constatados neste trabalho, por sua vez, fazem com que o desempenho do estudante diminua, interferindo em seu desenvolvimento e acarretando consequências para sua formação e atuação futura ${ }^{(5,19)}$.
Desta forma, as investigações sobre ansiedade em estudantes são de extrema importância e relevância nos meios científicos e assistenciais. Também se torna importante, pois o estudante percebendo a interferência negativa da ansiedade em seu desempenho, busca a ajuda profissional, muitas vezes, recorrendo ao uso de medicamentos para seu controle, e neste caso é importante que este seja feito de forma segura e que o estudante tenha conhecimento acerca do fármaco que utiliza $^{(10)}$.

Dos 308 estudantes que participaram do estudo, $16 \%$ usam ou já usaram algum tipo de ansiolítico, sendo o diazepam o fármaco mais utilizado. Estudos demonstraram como é comum e frequentemente indevido o uso de benzodiazepínicos entre estudantes de enfermagem, destacando as causas e os prejuízos decorrentes de sua terapia(8-10). A administração prolongada de benzodiazepínicos, mesmo em doses baixas, induz a prejuízos persistentes nas funções cognitivas e psicomotoras $^{(20)}$. Por se tratar de estudantes, este é um fato extremamente preocupante e que se relaciona aos efeitos colaterais do medicamento. Estes estão vinculados à depressão do sistema nervoso central causada pela droga e que podem levar à diminuição da atividade psicomotora, prejuízo na memória, desinibição paradoxal, tolerância, dependência e potencialização do efeito depressor pela interação com outras drogas depressoras, principalmente o álcool(18,20).

A orientação médica relacionada ao uso de ansiolíticos é um fator relevante para minimizar a incidência dos efeitos colaterais ${ }^{(21)}$, porém o presente estudo constatou que apesar de a maioria dos estudantes (75\%) ter sido orientada, 44\% deles ainda apresentaram dúvidas no que diz respeito principalmente à interação com outros fármacos, efeitos colaterais e se o medicamento causa dependência. Isto pode ser devido ao estudante não ter se lembrado da orientação ou não ter valorizado o que o médico orientou ou, ainda, a ênfase dada pelo médico para os cuidados com o uso dos benzodiazepínicos foi insuficiente ${ }^{(13)}$.

Foi constatado que $27 \%$ dos estudantes já aumentaram a dose do medicamento sem consultar o médico, sendo que todos os que possuíam ansiedade grave de acordo com o BAl o fizeram podendo, desta forma, ocorrer uma potencialização do efeito depressor da droga. Por outro lado, um fato que chamou atenção foi que $46 \%$ dos estudantes já interromperam o tratamento 
também sem consultar o médico, e se sabe que esta interrupção deve ser feita de maneira gradual para prevenir os sintomas de abstinência.

Em relação ao uso do álcool, $31 \%$ referiram ingerir concomitantemente ao uso do benzodiapínico, sendo que $19 \%$ o fazem aos finais de semana. Cabe destacar que a intoxicação resultante do sinergismo do efeito depressor causado pela interação de benzodiazepínicos e álcool pode ser grave e fatal, mesmo que o indivíduo o utilize socialmente ${ }^{(11)}$. Da mesma forma, $60 \%$ dos estudantes utilizam outras medicações, especialmente os antidepressivos como a fluoxetina, que também pode promover interação farmacológica importante.

\section{CONCLUSÃO}

Este estudo traz contribuições em relação à temática abordada, ao demonstrar a presença de diferentes níveis de ansiedade em estudantes de enfermagem, com alta porcentagem de estudantes com depressão moderada e grave. Constatou-se, ainda, que $16 \%$ dos estudantes utilizam ou já utilizaram algum medicamento ansiolítico, sendo o diazepam o mais frequentemente utilizado. Mesmo tendo recebido orientação, a maioria dos usuários de benzodiazepínicos referiram possuir dúvidas sobre o medicamento especialmente no que diz respeito à interação com outros fármacos, efeitos colaterais e seu

\section{REFERÊNCIAS}

1. Ferreira CL, Almondes KM, Braga LP, Mata ANS, Lemos CA, Maia EMC. Universidade, contexto ansiogênico? Avaliação de traço e estado de ansiedade em estudantes do ciclo básico. Cien saúde colet 2009;14(3):973-981.

2. Oliveira MA, Duarte AMM. Controle de respostas de ansiedade em universitários em situações de exposições orais. Rev bras ter comport Cogn 2004;6(2):183-200.

3. Peuker AC, Fogaça J, Bizarro L. Expectativas e beber problemático entre universitários. Psicologia: Teoria e Pesquisa 2006;22(2):193-200.

4. Dehghan-nayeri N, Adib-Hajbaghery M. Effects of progressive relaxation on anxiety and quality of life in female students: $A$ non-randomized controlled trial. Alternat Complement Ther 2011;19:194-200.

5. Melincavage SM. Student nurses' experiences of anxiety in the clinical setting. Nurse Educ Today 2011;31:785-789.

6. Oliveira KL, Santos AAA, Cruvinel M, Neri AL. Relação entre Ansiedade, Depressão e Desesperança entre Grupos de Idosos. Psicologia em Estudo 2006;11(2):351-359.

7. Lima MGR, Nietsche EA, Teixeira JA Reflexos da formação acadêmica na percepção do morrer e da morte por enfermeiros. Rev Eletr Enf [Internet] 2012; 14(1):181-8.

8. Picolotto E, Libardoni LFC, Migott AMB, Geib LTC. Prevalence and factor associated with psychoactives substances consumption for academics of Nursing of the University of Passo Fundo. Ciên saúde colet 2010;15(3):645-654. potencial para causar dependência. A presença de dúvidas em relação à terapia foi evidenciada pelo fato de um grande número de estudantes interromperem o tratamento e aumentar a dose do fármaco sem consulta médica.

Medidas que estimulem o uso racional de ansiolíticos devem ser adotadas assim como a orientação medicamentosa deve ser efetivada, visando minimizar riscos aos usuários destes fármacos, objetivando alcançar êxito com a terapia. É fundamental a adesão correta ao tratamento, sem interrupções ou aumento da dose, fatores estes, que foram identificados no presente estudo. Com esse tipo de abordagem, é possível minimizar os efeitos colaterais, evitando o desenvolvimento de dependência, abstinência, abuso e possíveis interações, direcionando o usuário para a utilização do fármaco de uma forma segura e principalmente efetiva.

Ressalta-se, ainda, a importância de adoção, pela instituição formadora, de medidas direcionadas aos fatores de risco para a ansiedade nos estudantes, de revisão das metodologias de ensino adotadas, bem como de estratégias que viabilizem a escuta e acolhimento aos mesmos, de modo a identificar a presença de ansiedade patológica e minimizar suas consequências.

9. Botti NCL, Lima AFD, Simões WMB. Uso de substâncias psicoativas entre acadêmicos de enfermagem da Universidade Católica de Minas Gerais. SMAD, Rev Eletrônica Saúde Mental Álcool Drog (Ed. port.) [online]. 2010;6(1):1-16.

10. Orlandi P, Noto AR. Uso indevido de benzodiazepínicos: um estudo com informantes-chave no município de São Paulo. Rev Latino-am Enfermagem 2005;13(número especial):896-902. 11. Auchewsk L, Andreatini R, Galduróz JCF, Lacerda RB. Avaliação da orientação médica sobre os efeitos colaterais de benzodiazepínicos. Rev Bras Psiquiatr 2004;26(1).

12. Cruz AV, Fulone I, Alcalá M, Fernandes AA, Montebelo MI, Lopes LC. Uso crônico de diazepam em idosos atendidos na rede pública em Tatuí-SP. Rev Ciênc Farm Básica Apl 2006;27(3):259267.

13. Beck AT, Epstein N, Brown G, Steer RA. An Inventory for Measuring Clinical Anxiety: Psychometric Properties. J Consult Clin Psychol 1988;56:893-897.

14. Andrade LHSG, Gorenstein C. Aspectos gerais das escalas de avaliação de ansiedade. Rev psiquiatr clín 1988;25(6).

15. Gultekin BK, Dereboy IF. Th e Prevalence of Social Phobia, and its Impact on Quality of Life, Academic Achievement, and Identity Formation in University Students. Turkish Journal of Psychiatry 2011;22(3):150-8.

16. Galindo SB, Moreno IM, Muñoz JG. Prevalencia de andiedad y Depressión em uma población de Estudiantes universitários: Factores acadêmicos y sociofamiliares asociados. Clínica y Salud 2009;20(2):177-187. 
17. Wagner GA, Andrade AG. Uso de álcool, tabaco e outras drogas entre estudantes universitários brasileiros. Rev psiquiatr clín [online] 2008;35(1).

18. Ortega-Pérez CA, Costa-Júnior ML, Vasters, GP. Perfil epidemiológico de la drogadicción en estudiantes universitários. Revista Latino-Americana de Enfermagem 2011;19:665-672.

19. Merrell, Kenneth. Helping Students overcome depression and anxiety: a pratical guide. $2^{\mathrm{a}}$ Edição; Nova lorque: Guilford press; 2008.

20. Castro GLG, Mendes CMM, Pedrini ACR, Gaspar DSM, Sousa FCF. Uso de Benzodiazepínicos como automedicação:

consequências do uso abusivo, dependência, farmacovigilância e farmacoepidemiologia. R. Interd 2013;6(1):112-123.

21. Tanskanen $P$, Airaksinen M, Tanskanen A, Enlund H.

Counselling patients on psichotropic medication: physicians opinions on the role of community pharmacists. Pharm World Sci 2000;22(2):59-61.

Artigo recebido em 13/06/2012.

Aprovado para publicação em 27/05/2013.

Artigo publicado em 30/09/2013. 